\title{
Indirekter Gegenentwurf zur Konzernverantwortungs- initiative - Haftungsnorm im Einklang mit der schweizerischen Tradition
}

\section{Franz Werro *}

Die Konzernverantwortungsinitiative wie der Gegenentwurf bauen mit der Geschäftsherrenhaftung auf einer allgegenwärtigen und in ihrer schweizerischen Ausgestaltung im internationalen Vergleich zurückhaltenden Haftungsnorm auf. Die Übertragung dieser Haftung auf Konzernverhältnisse folgt sodann einem klaren in- wie ausländischen Trend. Beide Vorlagen erweitern hingegen den geltenden Haftungsrahmen auch im vorliegend menschenrechtlichen Zusammenhang nicht, sondern konkretisieren ihn lediglich. Die im Gegenentwurf verankerte Haftungsregelung fällt zudem auffallend einschränkend aus. Zu nennen ist neben dem Entscheid für den unternehmerischen Entlastungsbeweis auch jener für einen eingeschränkten Kontrollbegriff. Die Haftungsnorm steht somit im Einklang mit der schweizerischen Rechtstradition. Dabei wählt die Schweiz eine im internationalen Vergleich zurückhaltende Lösung.

I. Einleitung

II. Beurteilung in fünf Grundthesen

1. Geschäftsherrenhaftung als im internationalen Vergleich allgegenwärtiges

Haftungskonzept

2. Artikel 55 OR als im internationalen Vergleich unternehmensfreundliche

Regelung.

3. Übertragbarkeit der Geschäftsherrenhaftung auf Konzernverhältnisse als in- und ausländischer Trend.

4. Anwendung dieses Haftungskonzepts im menschenrechtlichen

Zusammenhang - bescheiden bleibt bescheiden

5. Gedanken zur konkreten Regelung - Zurückhaltung nicht übertreiben .......438

III. Zusammenfassung

Zitiervorschlag: Franz Werro, Indirekter Gegenentwurf zur Konzernverantwortungsinitiative - Haftungsnorm im Einklang mit der schweizerischen Tradition, in: sui-generis 2018, S. 428

URL: $\quad$ sui-generis.ch/85

DOI: $\quad$ https://doi.org/10.21257/sg.85

* Prof. Dr. iur. Franz Werro (Franz.Werro[at]unifr.ch), Professor für Obligationenrecht und Europäisches Privatrecht, Universität Fribourg und Georgetown University Law Center (Washington, D.C.). Der Autor dieses Artikels war zur vorliegenden Thematik als Experte zur Anhörung der Rechtskommission des Ständerates vom 21. August 2018 eingeladen. Der vorliegende Beitrag gibt seine persönliche Auffassung wieder. Er dankt Frau Dipl. iur. Carola Göhlich, MLE, Assistentin an der Universität Fribourg, für ihre wertvolle Hilfe bei der sprachlichen Kontrolle des Manuskripts. 


\section{Einleitung}

1 Im Jahr 2015 lancierten rund 70 Nichtregierungsorganisationen eine eidgenössische Volksinitiative «Für verantwortungsvolle Unternehmen - zum Schutz von Mensch und Umwelt» (kurz: Konzernverantwortungsinitiative) ${ }^{1}$. Im Jahr 2016 wurde diese mit rund 120'ooo gültigen Unterschriften eingereicht ${ }^{2}$. In Nachachtung internationaler Leitlinien verfolgt die Initiative das doppelte Ziel, den internationalen Menschenrechtsund Umweltschutz bei wirtschaftlichen Aktivitäten mit Auslandsbezug zu stärken und dabei für mehr Rechtssicherheit zu sorgen. Der rechtliche Grundrahmen besteht darin, den Schweizer Unternehmen für ihre Auslandstätigkeiten eine Sorgfaltsprüfungspflicht aufzuerlegen und sie bei einer Pflichtverletzung in beschränktem Umfang auch haftbar zu machen. 3

2 Im Juni 2018 stimmte der Nationalrat einem indirekten Gegenentwurf (in der Folge: Gegenentwurf) seiner Rechtskommission mit 121 zu 73 Stimmen bei 2 Enthaltungen $\mathrm{zu}^{4}$. Zurzeit (Herbst/ Winter 2018) berät diesen die Rechtskommission des Ständerates. Der Gegenentwurf stützt sich auf dasselbe Grund-

1 Vorprüfung der Eidgenössischen Volksinitiative «Für verantwortungsvolle Unternehmen - zum Schutz von Mensch und Umwelt» (BBl 2015 3245).

2 Zustandekommen der Eidgenössischen Volksinitiative «Für verantwortungsvolle Unternehmen zum Schutz von Mensch und Umwelt» (BBl 2016 8107).

3 Zum Ganzen vgl. Art. 101a E-BV; zu den Erläuterungen der Initiative vgl. Verein Konzernverantwortungsinitiative (Hrsg.), Erläuterungen zur Eidgenössischen Volksinitiative «Für verantwortungsvolle Unternehmen - zum Schutz von Mensch und Umwelt», 2017, S. $10 \mathrm{ff}$.

4 SDA-Meldung vom 14. Juni 2018: Nationalrat will Konzerne in die Verantwortung nehmen. konzept wie die Initiative, enthält aber bedeutende Einschränkungen. So sind grundsätzlich nur Unternehmen ab einer bestimmten Grösse erfasst, und auch die Haftungsregelung ist enger gefasst als jene der Initiative (dazu im Anschluss). 5

3 Beide Vorlagen stützen ihre Haftungsregelung auf die Geschäftsherrenhaftung. Art. 55 Abs. $1^{\text {bis }}$ E-OR des Gegenentwurfs sieht im Einzelnen vor, dass nach den Grundsätzen der Geschäftsherrenhaftung ebenso Unternehmen haften, die nach Gesetz zur Einhaltung der Bestimmungen zum Schutz der Menschenrechte und der Umwelt auch im Ausland verpflichtet sind. In Anlehnung an den bestehenden Art. 55 Abs. 1 OR $^{6}$ haften sie «für den Schaden, den durch sie tatsächlich kontrollierte Unternehmen in Ausübung ihrer dienstlichen oder geschäftlichen Verrichtungen durch Verletzung der Bestimmungen zum Schutz der Menschenrechte und der Umwelt an Leib und Leben oder Eigentum im Ausland verursacht haben.» Die Unternehmen haften

5 Für die einzelnen Bestimmungen des Gegenentwurfs vgl. zur Übersicht die Anträge der Kommission für Rechtsfragen des Nationalrates [RK-N] vom 4. Mai 2018 zur eidgenössischen Volksinitiative «Für verantwortungsvolle Unternehmen zum Schutz von Mensch und Umwelt»: indirekter Gegenentwurf, die so unverändert vom Nationalrat verabschiedet wurden; zudem Zusatzbericht der Kommission für Rechtsfragen [RK-N] vom 18. Mai 2018 zu den Anträgen der Kommission für einen indirekten Gegenentwurf zur Volksinitiative «Für verantwortungsvolle Unternehmen zum Schutz von Mensch und Umwelt» im Rahmen der Revision des Aktienrechts (in der Folge: Kommissionsbericht).

6 Dieser lauter wie folgt: «Der Geschäftsherr haftet für den Schaden, den sein Arbeitnehmer oder andere Hilfspersonen in Ausübung ihrer dienstlichen oder geschäftlichen Verrichtungen verursacht haben, wenn er nicht nachweist, dass er alle nach den Umständen gebotene Sorgfalt angewendet hat, um einen Schaden dieser Art zu verhüten, oder dass der Schaden auch bei Anwendung dieser Sorgfalt eingetreten wäre.» 
nicht, «wenn sie nachweisen, dass sie die durch das Gesetz von ihnen geforderten Massnahmen zum Schutz der Menschenrechte und der Umwelt getroffen haben, um einen Schaden dieser Art zu verhüten[.]» Sie haften auch dann nicht, wenn sie nachweisen, dass sie «nicht auf das Verhalten des kontrollierten Unternehmens, in dessen Zusammenhang die geltend gemachten Rechtsverletzungen stehen, Einfluss nehmen konnten.»7

Soweit ersichtlich haben sich bislang nur vereinzelt Autoren mit haftpflichtrechtlicher Spezialisierung mit der vorstehenden Haftungsregelung auseinandergesetzt. ${ }^{8}$ Das überrascht: Denn die Haftung ist ein zentraler und gleichzeitig der politisch umstrittenste Teil der Vorlage. Angesichts der grenzüberschreitenden Ausstrahlung dieser Regelung und vor dem Erfahrungshintergrund als Inhaber eines Lehrstuhls für Privatrecht mit betont rechtsvergleichender Ausrichtung gehe ich hier den Grundfragen nach, ob die Haftungsregelung im internationalen Vergleich weit geht oder eingeschränkt ist und ob die Regelung den in der Schweiz bestehenden Haftungsrahmen

Art. 55 Abs. $1^{\text {bis }}$ E-OR des Gesetzesentwurfs (Fn. 5).

8 Vgl. für eine Einführung die Literaturhinweise bei Gregor Geisser, Die Konzernverantwortungsinitiative - Darstellung, rechtliche Würdigung und mögliche Umsetzung, AJP 8/2017, S. 943 ff. (953); vgl. auch Lukas Handschin, Konzernverantwortungsinitiative - Gesellschaftsrechtliche Aspekte, AJP 8/2017, S. 998 ff. (der trotz Titel auch haftpflichtrechtliche Aspekte aufgreift, die sich allerdings auf die Initiative beschränken und den damals noch nicht bestehenden Gesetzesentwurf nicht beinhalten). Auch nach jenen Publikationen ist die haftpflichtrechtliche Auseinandersetzung mit dieser Thematik kaum reicher geworden. dabei erweitert oder präzisiert. 9 Meine Beurteilung beinhaltet fünf Grundthesen.

\section{Beurteilung in fünf Grundthesen}

5 Die Betrachtung folgt einem schrittweisen Vorgehen: Sie beginnt mit der internationalen Verbreitung der Geschäftsherrenhaftung, führt über die Ausgestaltung von Art. 55 OR im Rechtsvergleich und behandelt schliesslich die Übertragbarkeit dieses Haftungskonzepts auf Konzernverhältnisse. Gestützt darauf folgt eine Beurteilung der Haftungsregelung im menschenrechtlichen Zusammenhang - zunächst im Allgemeinen und dann mit einem Ausblick auf den konkreten Gesetzesentwurf.

\section{Geschäftsherrenhaftung als im internationalen Vergleich allgegenwärtiges Haftungskonzept}

6 Sämtliche der mir geläufigen Rechtsordnungen kennen eine Geschäftsherrenhaftung oder funktional vergleichbare Institute. ${ }^{10}$ Diesen Haftungsnormen liegen primär die folgenden Rechtfertigungen einer Schadensabwälzung zugrunde: Wer

$9 \overline{\text { Für eine Einordnung aus Sicht des Internationa- }}$ len Privatrechts und Zivilprozessrechts vgl. u.a. die Quellen bei Geisser (Fn. 8), S. 946 ff. und $963 \mathrm{ff}$.

10 Für einen Überblick Paula Giliker, Vicarious Liability or Liability for the Acts of Others in Tort A comparative perspective, Journal of European Tort Law 2011, S. 31 ff.; Gerhard Wagner, Vicarious Liability, in: Arthur Hartkamp u.a. (Hrsg.), Towards a European Civil Code, 4. Aufl., The Hague u.a. 2011, S. 903 ff.; weiter auch Franz Werro/Vernon Palmer/Anne-Catherine Hahn, in: Franz Werro/Vernon Palmer (Hrsg.), The Boundaries of Strict Liability in European Tort Law, The Common Core of European Private Law, Bern u.a. 2004, S. 156 ff.; Franz Werro/ Erdem Büyüksagis, The Bounds between Negligence and Strict Liability, in: Mauro Bussani/ Anthony J. Sebok (Hrsg.), Comparative Tort Law: Global Perspectives, Cheltenham 2015, S. 218. 
eine andere Person kontrolliert, soll die Kontrolle über diese Personen auch zur Verhinderung von Schädigungen Dritter nutzen (sog. respondeat superior). Wer aus der Tätigkeit eines Anderen wirtschaftlichen Nutzen zieht, soll auch die damit verbundenen Risiken von Schädigungen Dritter tragen.11

7 Allen Haftungsnormen ist dabei gemein, dass sie (1) eine spezifische Beziehung, typischerweise ein Subordinations- bzw. Kontrollverhältnis, zwischen der unmittelbar schädigenden Person und der haftpflichtrechtlich mitverantwortlichen Person, (2) eine auf Sorgfaltsverletzung beruhende Schädigung durch die kontrollierte Person und (3) einen funktionalen Zusammenhang zwischen der Schädigung und der geschäftlichen Verrichtung für die kontrollierende Person voraussetzen. ${ }^{12}$

8 Auch der schweizerische Art. 55 OR folgt dieser Grundstruktur. Nicht zuletzt wegen ihrer bestechenden ratio legis und den klaren Haftungsvoraussetzungen ist diese Norm, wie auch in anderen Staaten,

11 Für die Schweiz vgl. bereits BGE 50 II 469 E. 2 S. 470; weitere Hinweise bei Franz Werro, La responsabilité civile, 3. Aufl., Bern 2017, Rz. 497; Walter Fellmann/Andrea Kottmann, Schweizerisches Haftpflichtrecht, Bd. I, Allgemeiner Teil sowie Haftung aus Verschulden und Persönlichkeitsverletzung, gewöhnliche Haftungen des OR, ZGB und PrHG, Bern 2012, Rz. 730 ff.; Christoph Müller, La responsabilité civile extracontractuelle, Bâle 2013, Rz. 266; vertiefend vgl. Martin Petrin, Fortentwicklung der Geschäftsherrenhaftung in der Schweiz, Zürich 2004, S. 21 ff.; rechtsvergleichend statt vieler Phillip Morgan, Vicarious liability for Group Companies: the Final Frontier of Vicarious liability?, Journal of Professional Negligence 4/2015, S. 289 f., m.w.H.; Siel Demeyere, Liability of a Mother Company for Its Subsidiary in French, Belgian, and English Law, European Review of Private Law 3/2015, S. 394 und 397, m.w.H.

12 So u.a. besonders prägnant Giliker (Fn. 10), S. 33 . immer wieder Modell gestanden für richterliche Fortbildungen, bis hin zu neuen gesetzlichen Regelungen im Zusammenhang mit Unternehmensrisiken. Prominentestes Beispiel einer solchen Rechtsentwicklung ist das Produktehaftpflichtgesetz. ${ }^{13}$ In die Reihe solcher Entwicklungen wäre auch die hier interessierende Vorlage einzuordnen, sollte sie dereinst in Kraft treten.

\section{Artikel 55 OR als im internationalen Vergleich unternehmensfreundliche Regelung}

9 Bei einem Vergleich der Geschäftsherrenhaftung mit analogen Haftungsnormen sind international drei Modelle vorherrschend: (a) das französische Modell, das sich an der Internalisierung von Geschäftsrisiken ohne individualisierbarem Fehlverhalten des Geschäftsherrn orientiert und sich entsprechend als «responsabilité sans faute» versteht, (b), das Modell des anglo-amerikanischen Rechtskreises, das unter dem breiten Dach einer «vicarious liability» ebenfalls als eine «strict liability» konzipiert ist, sowie (c) das von der Schweiz übernommene deutsche Modell, das sich als einfache Kausalhaftung nach wie vor am Grundsatz der mangelnden Sorgfalt des Geschäftsherrn orientiert. ${ }^{14}$

13 Vgl. BGE 110 II 456 («Schachtrahmen»-Fall) mit der Entwicklung bis hin zum Bundesgesetz vom 18. Juni 1993 über die Produktehaftpflicht (PrHG; SR 221.112.944); dazu vgl. Heinz Rey/ Isabelle Wildhaber, Ausservertragliches Haftpflichtrecht, 5. Aufl., Zürich u.a. 2018, Rz. 1117 ff.; Müller (Fn. 11), Rz. 426; zur verbleibenden Bedeutung von Art. 55 OR als Grundnorm etwa auch Werro (Fn. 11), Rz. 499 f.

14 Vgl. für eine Übersicht Wagner (Fn. 10), S. 903 ff.; Giliker (Fn. 10), S. 32 ff.; auch Jaap Spier (Hrsg.), Unification of Tort Law: Liability for Damage caused by Others, The Hague u.a. 2003, S. 3 ff. 
Im Kern dieser Unterscheidung geht es um die Frage, ob der ins Recht gefassten Person (hier dem inländischen Unternehmen) eine Haftungsbefreiung durch Sorgfaltsbeweis offen steht oder nicht. Bei der schweizerischen Lösung ist ein solcher Befreiungsbeweis vorgesehen. Bei den schärferen Haftungen, die neben dem anglo-amerikanischen Rechtskreis gerade auch in vielen kontinentaleuropäischen Ländern wie z.B. Frankreich, Belgien, Luxembourg oder Italien vorkommen, fällt ein solcher hingegen weg. Sind die vorstehenden drei Haftungsvoraussetzungen erfüllt, haftet die beklagte Person ohne weiteres; eine Sorgfaltsverletzung durch den Geschäftsherrn ist im Grundsatz nicht vorausgesetzt. ${ }^{15}$

In dieser Einordnung befindet sich die Schweiz betreffend Schärfegrad somit überspitzt gesagt im letzten Drittel der Staatengemeinschaft. Angesichts der von einer Sorgfaltsverletzung zunehmend unabhängiger verstandenen Geschäftsherrenhaftung in Deutschland bewegt sich die Schweiz europäisch gar in Richtung Schlusslicht. ${ }^{16}$ So hat sich auch hierzulande die Meinung durchgesetzt, dass der Sorgfaltsbeweis ein Relikt einer stark am persönlichen Verhalten der Geschäftsherrin bzw. des Geschäftsherrn angelehnten Konzeption ist. Diese passt schlecht zur heutigen Gliederung der Unternehmungen, die sich durch eine starke Konzentration der Entscheidungsbefugnisse und eine spezialisierte Arbeitstei-

15 Vgl. prägnant Vito Roberto, Haftpflichtrecht, 2. Aufl., Bern 2018, Rz. 08.59; eingehender u.a. Giliker (Fn. 10), S. 31 ff., 37 f.; Demeyere (Fn. 11), S. 407; Morgan (Fn. 11), S. 276; Spier (Fn. 14), S. $63 \mathrm{ff}$.

16 Vgl. zur zusehends strengeren Praxis in Deutschland z.B. Nachweise bei Giliker (Fn. 10), S. 36 f.; Wagner (Fn. 10), S. 911. lung - ohne klar individualisierbarem Fehlverhalten - auszeichnen. ${ }^{17}$ Zumindest Ansätze einer etwas sorgfaltsunabhängigeren Organisationshaftung tragen das Projekt «OR 2020» ${ }^{18}$ und der Vorentwurf eines Bundesgesetzes zur Revision und Vereinheitlichung des Haftpflichtrechts («Widmer/Wessner»)19. Zweimal hat der Bundesrat diese Verschärfung allerdings nicht weiterverfolgt. ${ }^{20}$

12 Indem die Konzernverantwortungsinitiative wie der Gegenentwurf dem beklagten Unternehmen nach wie vor einen Sorgfaltsbeweis belassen, bauen beide Vorlagen folglich auf einer herkömmlichen und wirtschaftsfreundlichen Ausgestaltung der Geschäftsherrenhaftung auf. Sie stützen sich zudem auf eine Regelung, die im internationalen Vergleich äusserst milde und bescheiden ausfällt.

$17 \overline{\text { So grundlegend schon Peter Jäggi ZSR 86/1967 }}$ II, Diskussionsvotum am Schweizer Juristentag, S. $754 \mathrm{ff}$.

18 Als informativen Einstieg dazu vgl. www.or2020.ch; Walter Fellman/Christoph Müller/Franz Werro, Kommentierung zu Art. 46-63, in: Claire Huguenin/Reto M. Hilty (Hrsg.), Schweizer Obligationenrecht 2020 - Entwurf für einen neuen allgemeinen Teil, Zürich 2013.

19 Im Vergleich mit den europäischen Entwicklungen vgl. Franz Werro, The Swiss Tort Reform: a Possible Model for Europe?, in: Mauro Bussani (Hrsg.), European Tort Law, Bern 2007, S. 81 ff.; zur Kritik am Sorgfaltsbeweis mit etwas anderer Begründung Roberto (Fn. 15), Rz. 08.60 ff.; vertiefend Oliver Waespi, Organisationshaftung, Bern 2005, passim.

20 Dass die Zeit für einen solchen Schritt hierzulande offenbar noch nicht reif ist, hat der Bundesrat unlängst bekräftigt (vgl. Bericht des Bundesrates vom 31. Januar 2018 - Modernisierung des Allgemeinen Teils des Schweizer Obligationenrechts in Erfüllung der Postulate [13.3217] Bischof und [13.3226] Caroni). Bereits den erwähnten Vorentwurf «Widmer/Wessner» hatte der Bundesrat nicht weiterverfolgt. 


\section{3. Übertragbarkeit der Geschäftsherren- haftung auf Konzernverhältnisse als in- und ausländischer Trend}

13 Zur punktuellen Relativierung von Haftungsschranken im Konzern gegenüber geschädigten Dritten - sei es allgemein oder gerade auch im vorliegenden $\mathrm{Zu}$ sammenhang - richtet die neuere Lehre ihren Blick zusehends auf die Geschäftsherrenhaftung oder vergleichbare Institute. ${ }^{21}$ In der internationalen Literatur gewinnt diese Diskussion v.a. unter den Titeln «vicarious liability» bzw. «liability for the acts of others» an Schärfe. So befürwortet etwa die Doktrin in England, Frankreich oder den Niederlanden aber auch in anderen Staaten mit zunehmender Deutlichkeit eine Übertragbarkeit der Geschäftsherrenhaftung oder vergleichbarer Institute auf Konzernverhältnisse. Dabei soll nicht unerwähnt bleiben, dass Klagen zur Anwendung dieser Ansätze bislang noch die Ausnahme bleiben. ${ }^{22}$

21 Dies in Abgrenzung zur Durchgriffshaftung, die als zu radikale Lösung zu Recht je länger je weniger von Bedeutung ist: dazu treffend Geisser (Fn. 8), S. 953 f.; Rolf H. Weber/Rainer Baisch, Liability of Parent Companies for Human Rights Violations of Subsidiaries, 2015, S. 688 ff.; und im anglo-amerikanischen Rechtskreis in letzter Differenzierung zu Recht auch vermehrt mit Abstand vom sog. «Duty-of-Care-Konzept» hin zur «vicarious liability» u.a. Morgan (Fn. 11), S. $276 \mathrm{ff}$.

${ }^{22} \mathrm{Zu}$ dieser Diskussion vgl. aus der reichen Literatur allgemein statt vieler Demeyere (Fn. 11), S. 385 ff.; Morgan (Fn. 11), S. 276 ff. und S. 289 ff. (v.a. in der Ausgestaltung als «enterprise liability»); Karl Hofstetter, Sachgerechte Haftungsregeln für multinationale Konzerne - Zur zivilrechtlichen Verantwortlichkeit von Muttergesellschaften im Kontext internationaler Märkte, Tübingen 1995, S. 119 ff. (S. 225 f.: Nachweise zu Deutschland mit Blick auf die Geschäftsherrenhaftung); und mit zusätzlich menschenrechtlichem Bezug: Weber/Baisch (Fn. 21), S. 685 ff.; Geisser (Fn. 8), S. 952 ff.; Liesbeth Enneking, Corporate liability for violations of human rights and the environment abroad: a comparative perspective, AJP 8/2017, S. 988 ff.; André Nollkaemper, Public International Law in Transna-
14 In der Schweiz steht die Diskussion an einem ähnlichen Punkt. Nach breit abgestützter und ganz vorherrschender Lehrmeinung soll Art. 55 OR auch dann anwendbar sein, wenn nicht natürliche Personen, sondern Unternehmen als juristische Personen Geschäftsherr und Hilfsperson sind (vgl. dazu den eindrücklichen Fussnotenkatalog).23 Eine Übertragung folgt der Art. 55 OR zugrundeliegenden Rechtfertigung, dass soweit herrschende Gesellschaften ihren Handlungsspielraum durch den Einsatz von abhängigen Gesellschaften ausweiten, sie

tional Litigation Against Multinational Corporations: Prospects and Problems in the Courts of Netherlands, in: Kamminga Menno T./Zia-Zarifi Sman (Hrsg.), Liability of Multinational Corporations under International Law, The Hague 2000, S. 265 ff.; Cees van Dam, Tort Law and Human Rights: Brothers in Arms - On the Role of Tort Law in the Area of Business and Human Rights, Journal of European Tort Law 2011, S. $221 \mathrm{ff}$,, $247 \mathrm{ff}$.

23 Vgl. Roland von Büren, Schweizerisches Privatrecht Bd. VIII/6, Der Konzern, 2. Aufl., Basel u.a. 2005, S. 202 ff.; Hofstetter (Fn. 22), S. 239 ff.; Lukas Handschin, Der Konzern im geltenden schweizerischen Privatrecht, Zürich 1994, S. 344 ff.; Karin Beyeler, Konzernleitung im schweizerischen Privatrecht, Zürich 2004, S. 272 ff.; Nina Sauerwein, La responsabilité de la société mère, Bern 2006, S. 89 ff.; Max AlbersSchönberg, Haftungsverhältnisse im Konzern, Zürich 1980, S. 174 ff.; Jean Nicolas Druey/Alexander Vogel, Das Schweizer Konzernrecht in der Praxis der Gerichte, Zürich 1999, S. 101; Gregor Geisser, Ausservertragliche Haftung privat tätiger Unternehmen für Menschenrechtsverletzungen bei internationalen Sachverhalten - Möglichkeiten und Grenzen der schweizerischen Zivilgerichtsbarkeit im Verhältnis von Völkerrecht und Internationalem Privatrecht, Zürich u.a. 2013, S. 494 ff. Im Grundsatz so auch von der Praxis bejaht: vgl. Urteil des Bundesgerichts (R. Inc. c. S. SA) vom 11. Juni 1992, in: SZW 1993, S. 308. Als soweit ersichtlich einzig kritische Stimmen im vorliegenden Zusammenhang, allerdings ohne Bezug zur vorstehenden Literatur, Peter Böckli/Christoph B. Bühler, Zur «Konzernverantwortungsinitiative» - Rechtliche Überlegungen zu den vier Forderungen der Eidgenössischen Volksinitiative «Für verantwortungsvolle Unternehmen von Mensch und Umwelt», Zürich u.a. 2018, S. 56 f. 
auch für die Handlungen in diesem erweiterten Handlungsbereich haftpflichtrechtlich mitverantwortlich sind (respondeat superior).24 Als Haftpflichtrechtler lasse ich mich in diesem rechtsgebietsübergreifenden Thema von der zitierten Konzernrechtslehre mit Nachdruck davon überzeugen, dass die von Art. 55 OR verlangte «ökonomischorganisatorische» 25 Subordination und die damit verbundene faktische Weisungsmacht zwischen Geschäftsherr und Hilfsperson in der Realität zunehmend straff geführter Konzerne zwischen Mutter- und Tochtergesellschaft typischerweise gegeben ist. ${ }^{26}$ In dieser Wirklichkeit kann es etwa zu den Konzernleitungsaufgaben gehören, den abhängigen Tochtergesellschaften operationelle Vorgaben zu Umsatz- und Ertragszielen oder zur Produktion zu machen. ${ }^{27}$

Mit der Formulierung «tatsächlich kontrollierte Unternehmen» in Art. 55 Abs. $1^{\text {bis }}$ E-OR entscheidet sich der indirekte Gegenentwurf - in Anlehnung an das im Konzernrecht an sich überkommene Leitungsprinzip - für ein enges Verständnis einer haftungsrelevanten Kontrolle. ${ }^{28}$ Ein solches vertritt etwa Roland von Büren: Nur wenn der herr-

24 Vgl. treffend Handschin (Fn. 23), S. 347.

25 Vgl. u.a. Rey/Wildhaber (Fn. 13), Rz. 1066, m.w.H.; Fellmann/Kottmann (Fn. 11), Rz. 739; Petrin (Fn. 11), S. 67 ff.

26 Beispielhaft für die vorstehende Literatur vgl. Handschin (Fn. 23), S. 349.

27 Vgl. etwa von Büren (Fn. 23), S. 57. Mischt sich die Konzernmutter (mittels ihrer Organe) darüber hinaus in die konkret schädigende Handlung ein, indem sie z.B. spezifische, die absolut geschützten Rechtsgüter Dritter verletzende Weisungen erteilt, wird sie gar zur Geschäftsführerin und wäre unter Umständen direkt aus Art. 41 OR haftbar; vgl. dazu Nachweise bei Geisser (Fn. 23), S. $493 \mathrm{f}$.

28 Dazu auch Kommissionsbericht (Fn. 5), Kap. 2.3.1. schenden Gesellschaft eine mit der Stellung einer Geschäftsherrin vergleichbare Möglichkeit zukommt, durch Beeinflussung der abhängigen Gesellschaft eine Schädigung Dritter zu vermeiden, ist die für eine Haftung nach Art. 55 OR erforderliche faktische Weisungsmacht gegeben. Das herrschende Unternehmen ist demnach dort, wo es eine einheitliche Leitung durchsetzt (oder eben «tatsächliche Kontrolle» ausübt), als Geschäftsherr zu qualifizieren. In Bereichen hingegen, welche nicht von der einheitlichen Leitung betroffen sind und wo das abhängige Unternehmen autonom handelt, kann diese Haftung nur ausnahmsweise und unter strengen Voraussetzungen, z.B. bei einer konkreten Weisungsvereinbarung, in Frage kommen. So in etwa dürfte das enge Verständnis des Gesetzesentwurfs auszulegen sein. Denkbar wäre es mit Lukas Handschin aber auch gewesen, auf das im Gesellschaftsrecht mittlerweile etablierte Kontrollprinzip abzustellen, wonach zwar die Leitungsmechanismen eingerichtet sein müssen, die Leitung aber nicht unbedingt effektiv wahrgenommen worden sein muss. ${ }^{29}$ Der Gegenentwurf enthält an dieser Stelle denn auch eine deutliche Einschränkung zur Initiative, welche eine Kontrolle «faktisch auch durch wirtschaftliche Machtausübung» genügen lässt. $3^{\circ}$ Soweit meine Ausführungen zur Kontrollvoraussetzung. Damit komme ich zum Sorgfaltsbeweis.

29 Handschin (Fn. 23), S. 349 und 353; im vorliegenden Verhältnis Ders. (Fn. 8), S. 1000 ff. (mit dem Hinweis auf das Kontrollprinzip bzw. die Konsolidierungspflicht nach Art. 963 OR als objektives und rechtssicheres Kriterium).

30 Vgl. Art. 101a Abs. 2 Bst. a E-BV; zum Verständnis dieser Formulierung vgl. auch Erläuterungen (Fn. 3), S. 43 f. 
Zur Weiterentwicklung der Geschäftsherrenhaftung unter dem Titel «sachgerechte[r] Haftungsregeln für multinationale Konzerne» ist die Stimme von Karl Hofstetter am einschlägigsten. In seiner gleichnamigen Habilitation hat er die haftungsrelevanten Sorgfaltskriterien bei Konzernverhältnissen wie folgt umrissen: «Der Sorgfaltsbeweis der Konzernmutter sollte sich auch im Zusammenhang mit der Geschäftsherrenhaftung am Begriff der ordnungsgemässen Konzerngeschäftsführung orientieren. Diesen gilt es spezifisch auf die [...] Schutzinteressen ausservertraglicher Tochtergläubiger auszurichten. Dabei ist der konkreten Ausgestaltung der Mutterkontrolle über die Tochtertätigkeiten und der Voraussehbarkeit eingetretener Risiken Rechnung zu tragen. Der Nachweis der drei klassischen curae [...] vermag aber grundsätzlich nicht zu genügen. $G e-$ fordert ist vielmehr Beweis dafür, dass alle konzernorganisatorischen Möglichkeiten ausgeschöpft wurden, um eine optimale Sicherheit der Tochtertätigkeiten zu gewährleisten (Konzernorganisationshaftung).»31

Bereits dem geltenden Recht von Art. 55 OR liegen demnach Konturen einer Konzernorganisationshaftung zugrunde. Eine Verantwortlichkeit des Mutterunternehmens für die schädigenden Handlungen ihrer Töchter nimmt dabei Abstand vom klassischen Verständnis einer natürlichen Person als Hilfsperson und den darauf zugeschnittenen drei curae der Sorgfalt in deren Auswahl, Instruktion und Überwachung. Die Verantwortlichkeit bezieht sich dagegen vornehmlich auf die vierte vom Bundesgericht entwi-

31 Hofstetter (Fn. 22), S. 243 f., m.w.H. (Hervorhebung hinzugefügt). ckelte curae, nämlich jene einer zweckmässigen Organisation. Eine dahingehende Organisationshaftung hat sich etwa in der Produktehaftpflicht verfestigt. $3^{2}$

18 Dementsprechend rege ich dazu an, die vorliegende Haftungsregelung in einer eigenen Bestimmung unterzubringen und nicht, wie im Gegenentwurf, im bestehenden Art. 55 OR zu integrieren. Eine neue und separate Bestimmung soll zwar weiterhin den Geist der Geschäftsherrenhaftung atmen, in funktionaler Umsetzung aber ihre konzernspezifische Ausgestaltung erhalten.

19 Als Zwischenfazit stelle ich fest, dass die Übertragung der Geschäftsherrenhaftung auf Konzernverhältnisse in adäquater Umsetzung sowohl in der Schweiz als auch im Ausland einem bereits im geltenden Recht angelegten, mehrheitsfähigen Trend entspricht. Die zu beurteilende Vorlage baut somit auf einer sachgerechten und bestehenden Rechtsgrundlage auf.

\section{Anwendung dieses Haftungs- konzepts im menschenrechtlichen Zusammenhang - bescheiden bleibt bescheiden}

20 Die Konzernverantwortungsinitiative wie der Gegenentwurf stützen sich nach den Gesagten auf eine adäquate Haftungsnorm. In der Ausgestaltung bleibt Art. 55 OR dabei wegen dem Entlastungsbeweis als herkömmlich-zurückhaltend zu bezeichnen. Im menschenrechtlichen $\mathrm{Zu}-$ sammenhang kann der Aufbau auf eine Geschäftsherrenhaftung, die dem beklag-

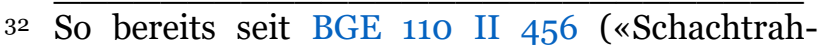
men»-Fall). 
ten Unternehmen nach wie vor solchen Entlastungsbeweis belässt, gleichwohl seine Berechtigung haben. So steht der entworfene Gesetzesrahmen inspiriert von den international anerkannten UNOLeitprinzipien für Wirtschaft und Menschenrechte (2011) auf zwei Beinen:33

1. Zunächst sieht er eine unternehmerische Sorgfaltsprüfungspflicht vor, die in voller Reichweite vorab in einer gesellschaftsrechtlichen Berichterstattungspflicht mündet. 34

2. Bei Verletzung dieser Sorgfaltsprüfungspflicht kennt die Vorlage zur staatlichen Durchsetzung und effektiven Wiedergutmachung sodann eine beschränkte Haftpflicht. 35

Die Verbindung dieser Sorgfaltsprüfungspflicht und Haftung erfolgt durch den erwähnten Entlastungsbeweis. Um sich von Haftungsrisiken freizuhalten, hat das Unternehmen die gesetzlich geforderte Sorgfalt walten zu lassen. Hat es diese Sorgfalt angewendet, kann es sich von der Haftung entlasten. Diese haftpflichtrechtliche Wirkung schafft für Unternehmen den Anreiz, der gesellschaftsrechtlich verankerten Sorgfaltsprüfungs-

33 Vgl. die vom UNO-Menschenrechtsrat am 21. März 2011 (UN Doc. A/HRC/17/31) verabschiedeten UNO-Leitprinzipien für Wirtschaft und Menschenreche, 2011. Für diesen Leitlinien mit Blick auf die Schweiz mitunter zugrunde liegende Schutzpflichten bei Handlungen von Schweizer Unternehmen (auch bei Auslandsaktivitäten) vgl. Evelyne Schmid, Exigences internationales de prendre des mesures législatives - La Suisse doitelle légiférer dans le domaine des «entreprises et droits humains»?, AJP 8/2017, S. 930 ff.; bezüglich Haftpflichtrecht vgl. Geisser (Fn. 23), Rz. $115 \mathrm{ff}$. und $203 \mathrm{ff}$.

$34 \mathrm{Vgl}$. UNO-Leitprinzipien (Fn. 33) 17 ff. mit Art. $716 \mathrm{a}^{\text {bis }}$ und Art. 961e E-OR des Gesetzesentwurfs (Fn. 5).

35 Vgl. UNO-Leitprinzipien (Fn. 33) 26 mit Art. 55

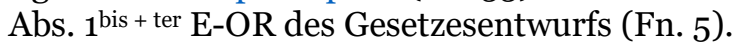

pflicht mit der nötigen Ernsthaftigkeit zu begegnen. Das geschickte Zusammenspiel von Gesellschafts- und Haftpflichtrecht kann insoweit betont präventiv wirken. ${ }^{36}$ Diese Korrelation bringt das UNO-Hochkommissariat für Menschenrechte im aktuellen Bericht «The relevance of human rights due diligence to determinations of corporate liability» wie folgt auf den Punkt: «Permitting a defense to liability based upon human rights due diligence activities could incentivize companies to meaningfully engage in such activities and have important preventative effect [...].»37

22 Gleichwohl vermag eine Bestimmung wie die vorgeschlagene, die dem Entlastungsbeweis verhaftet bleibt, das vorstehend skizzierte Gewand einer althergebrachten und vergleichsweise harmlosen Regelung nicht abzustreifen. ${ }^{38}$ Bescheiden bleibt eben bescheiden. Ob die Schweiz dieser sehr unternehmensfreundlichen Zurückhaltung auch im vorliegenden Zusammenhang treu bleiben will, ist letztlich eine politische Frage.

23 Meine weiteren Überlegungen gehen sodann der Frage nach, wie der Sorgfaltsmassstab ausgestaltet sein soll: Art. $716 \mathrm{a}^{\text {bis }} \mathrm{E}-\mathrm{OR}$ (in Verbindung mit Art.

$36 \overline{\text { Statt vieler vgl. Van Dam (Fn. 22), S. } 221 \text { ff.; Eu- }}$ roparat, Recommendation CM/Rec(2016)3 of the Committee of Ministers to member States on human rights and business, 2. März 2016, Ziff. $32 \mathrm{ff}$.

37 Report of the United Nations High Commissioner for Human Rights (OHCHR), Improving accountability and access to remedy for victims of business-related human rights abuse: The relevance of human rights due diligence to determinations of corporate liability, 1. Juni 2018, UN Doc. A/HRC/38/20/add.2, Ziff. 29.

38 Dessen ist sich bei rechtsvergleichender Betrachtung offenbar auch das UNO-Hochkommissariat für Menschenrechte bewusst: vgl. OHCHR (Fn. 37), Ziff. 26 und 29 (ganzer Absatz). 
55 Abs. $1^{\text {bis }}$ E-OR) leitet die Unternehmen zunächst zum Dreischritt an, (1) Auswirkungen der Geschäftstätigkeit auf Menschenrechte und Umwelt $\mathrm{zu}$ ermitteln und einzuschätzen, (2) die nötigen Massnahmen umzusetzen und zu überwachen sowie (3) darüber zu berichten. Diese Trilogie stützt sich auf die international anerkannten, sehr gut strukturierten und praxisnahen UNO-Leitprinzipien für Wirtschaft und Menschenrechte.39 Konzernspezifisch haben gewichtige Stimmen in der Lehre gestützt auf einschlägiges Rechtsmaterial (darunter die OECD-Leitsätze für multinationale Unternehmen)40 dabei die folgende Sorgfalts-Formel entworfen: Die unter den gegebenen Umständen gebotene Sorgfalt im Konzern ergibt sich letztlich aus einer zweckmässigen, dem Verhältnis von Kontrolle, Risiken und Mitteln entsprechende Kompetenzaufteilung innerhalb der Unternehmensgruppe, die organisatorisch darauf abzielt, menschenrechtliche Beaufsichtigungslücken $\mathrm{zu}$ vermeiden. 41

In dezentralen Konzernbereichen geht es demnach seitens der Konzernleitung um ein sorgfältiges Monitoring der konzernweiten Gesamtstruktur verbunden mit einer hinreichenden finanziellen, personellen und technischen Ausstattung der kontrollierten Gesellschaft und weniger um die Überwachung jedes Einzelrisikos.

39 Vgl. Fn. 30. Dazu Kommissionsbericht (Fn. 5), S. 5 .

40 Vgl. die OECD-Leitsätze für multinationale Unternehmen, 2011. Dazu aktuell auch die detailliertere OECD Due Diligence Guidance for Responsible Business Conduct, 2018. Für mögliche rechtliche Wirkungen dieser Leitsätze vgl. bereits Hans W. Baade, The Legal Effect of Codes of Conduct for Multinational Enterprises, German Yearbook of International Law 1979, S. $11 \mathrm{ff}$.

41 Vgl. die Verweise in der nachstehenden Fn.
Anders liegen die Dinge in zentralistisch organisierten Bereichen des Konzerns, in welchen die Mutter die Tochter im Detail kontrolliert und daher auch in der Lage ist, die weisungsmässige Abwicklung der Geschäfte zu überprüfen. ${ }^{42}$

25 Diese Ausführungen sind vielversprechend und lohnen einer näheren Betrachtung. Im vorliegenden Beitrag bleibt kein Raum für eine solche Vertiefung. Für meine Grundthese genügt die Feststellung, dass eine Konkretisierung der gebotenen Sorgfalt in die vorskizzierte Richtung gehen kann und muss, um der in Art. 55 OR angelegten Konzernorganisationshaftung weitere Konturen zu verleihen. So gesehen bildet die diskutierte Vorlage einen gesetzlichen Grundrahmen, der die spezifisch menschenrechtlichen Aufgaben der Leitungsorgane im Konzern weiter klärt, aber auch deren Grenzen aufzeigt. Eine solche Gesetzgebung wäre somit der Rechtssicherheit dienlich. 43

$42 \overline{\text { Vgl. aus dem Kreis dieser gewichtigen Stimmen }}$ u.a. Handschin (Fn. 23), S. 111 und S. 352 f. (allgemein); OECD-Leitsätze (Fn. 40), Erläuterungen zu den allgemeinen Grundsätzen, Ziff. 9 (zum Gesamtmonitoring); Peter Forstmoser, Schutz der Menschenrechte - eine Pflicht für multinationale Unternehmen?, in: Angela Cavallo u.a. (Hrsg.), Liber amicorum für Andreas Donatsch, Zürich u.a., 2012, S. 715 (zur Organisationspflicht) und S. 720; Ders., Corporate Social Responsibility, eine (neue) Rechtspflicht für Publikumsgesellschaften?, in: Robert Waldburger u.a. (Hrsg.), Festschrift für Peter Nobel zum 70. Geburtstag, Bern 2015, S. 157 ff. und S. 175; von Büren (Fn. 23), S. 203 (zur haftungsrelevanten Aufsichtspflicht der Konzernmutter); Hofstetter (Fn. 22), S. 243 (Sorgfalts-Formel); Rey/ Wildhaber (Fn. 13), Rz. 1103 (zur Vermeidung von Beaufsichtigungslücken); in gesamthaftem Zusammenzug vgl. Geisser (Fn. 8), S. 957 f. (mit Anwendungsbeispielen).

43 Als Potenzial und Empfehlung in diese Richtung namentlich für civil-law-Staaten [wie der Schweiz] vgl. auch OHCHR (Fn. 37), Ziff. 24 und Annex I, Policy objective 14.2-3; aus der Lehre 
Nach diesen Erkenntnissen muss ich als Haftpflichtrechtslehrer den mitunter schrillen und politisch anmutenden Stimmen entschieden widersprechen, die ins Feld führen, die Konzernverantwortungsinitiative wie der indirekte Gegenentwurf schafften eine vollkommen neue Haftungsgrundlage, was zu Rechtsunsicherheit und einem massiv höheren Haftungsrisiko führe.44 Die vorgesehene Haftungsregelung erweitert nach dem Gesagten den Haftungsrahmen nicht, sondern präzisiert ihn lediglich. Und wer die Haftungsregelung mit Blick auf die angesprochenen Menschenrechts- und Umweltgüter als ausufernd erachtet, überliest in Art. 55 Abs. $1^{\text {bis }}$ E-OR das gesetzliche Erfordernis eines Schadens an «Leib und Leben oder Eigentum». Mit diesem rechtstechnisch wohl besser zu bezeichnenden Personen- und Sachschaden bleibt die eng verstandene Thematik haftpflichtrechtlich gefasst. Nur soweit die Verletzung der menschen- und umweltrechtlichen Sorgfaltsprüfungspflicht zu derart handfesten Schädigungen an privatrechtlich geschützten Gütern führt, ist sie haftungsrelevant. Die Regelung führt dementsprechend auch nicht etwa $\mathrm{zu}$ einem neuen internationalen Umwelthaftungsrecht.

Nicolas Bueno, La responsabilité des entreprises de respecter les droits de l'homme - État de la pratique suisse, AJP 8/2017, S. 1023 f.; Enneking (Fn. 22), S. 996; Geisser (Fn. 8), S. 962; innerhalb der von SKMR/SIR (Hrsg.), mit Christine Kaufmann und Lukas Heckendorn Urscheler, in ihrer Wiedergutmachungs-Studie [Access to Remedy - Study commissioned by the FDFA with view to fulfilling Postulate 14.3663], 2017, Executive Summary, S. 10 und Bericht, S. 131 ff., dargelegten Optionen, entspricht dieses Vorgehen dem vermittelnden Szenario C: Klärung statt Erweiterung.

44 Vgl. z.B. Felix Ehrat, «Die Initiative ist eine Mogelpackung», NZZ online vom 8. November 2016.
27 Ausfransende Tendenzen dieser Haftungsregelung sind entgegen gegenteiliger Bedenken 45 auch insoweit keine auszumachen, als die Bestimmung nicht über den Konzern hinausgeht und als solche keine Haftungswirkung in die Zulieferkette hat. In Art. 55 Abs. $1^{\text {ter }}$ E-OR ist dazu ausdrücklich vermerkt, dass «ein Unternehmen [...] ein anderes Unternehmen nicht allein deswegen [kontrolliert], weil dieses von jenem wirtschaftlich abhängt». Eine haftungsrelevante Kontrolle ist damit ausserhalb rechtlicher und im Konzern auch tatsächlich ausgeübter Kontrolle ausgeschlossen. Überschiessende Tendenzen einer Haftung hin zu Schädigungen durch Lieferanten, wie sie etwa in der französischen «Loi relative au devoir de vigilance des sociétés mères» im menschenrechtlichen Zusammenhang im Begriff der «relation commerciale établie» angelegt sind, 46 beinhaltet der Gegenentwurf nicht. 47

\section{Gedanken zur konkreten Regelung - Zurückhaltung nicht übertreiben}

28 Die Rechtskommission des Ständerates ist im Herbst 2018 auf den indirekten Gegenentwurf mit 9 gegen 2 Stimmen bei einer Enthaltung eingetreten. Gleichzeitig hat sie in ihrer Medienmitteilung vom 17. Oktober 2018 verlauten lassen, die Arbeiten am vom Nationalrat verab-

45 Vgl. etwa Ehrat (Fn. 44).

46 Neue Artikel L225-102-4, L225-102-5 im Code de commerce, eingeführt durch Loi ${ }^{\circ}$ 2017-399 relative au devoir de vigilance des sociétés mères et des entreprises donneuses d'ordre vom 27. März 2017, in Kraft seit dem 29. März 2017. In Auseinandersetzung mit der konkreten Frage vgl. Stéphane Brabant u.a., The Vigilance Plan - Cornerstone of the Law of the Corporate Duty of Vigilance, Revue Internationale de la Compliance, Suppl., 2017, S. 2 f.

47 Vgl. explizit auch Kommissionsbericht (Fn. 5), Kap. 2.3.1, dritter Absatz. 
schiedeten Text weiterzuführen ${ }^{4}$. Dieses Vorgehen ist aus rechtlicher Sicht insoweit zu begrüssen, als mit der Fortführung der Arbeiten legistische Präzisierungen gemeint sind. Die eingangs wiedergegebene Bestimmung von Art. 55 Abs. $1^{\text {bis }}$ E-OR ist lang, als solche schwer lesbar und mitunter redundant. 49 Kaum nachvollziehbar ist dabei, dass das gerade im vorliegenden Zusammenhang eminent wichtige Wort «Sorgfalt» in der deutsch- und französischsprachigen Textfassung fehlt. Auch empfehle ich aus den erwähnten Gründen (vgl. Kap. II.3), die Haftungsregelung strukturell vom bestehenden Art. 55 OR abzukoppeln und die gehaltvollen Aussagen von Art. 55 Abs. $1^{\text {bis }}$ E-OR stattdessen in eine eigenständige rechtsformneutrale Norm zu giessen. Im vorliegenden Grundsatzbeitrag gehe ich legistischen Fragen nicht weiter nach und gehe davon aus, dass die zuständige Rechtskommission diese erfolgreich bereinigt.

Einem Ansinnen nach Präzisierung kann ich aus haftpflichtrechtlicher Sicht aber dann nicht folgen, wenn damit weitere materielle Einschränkungen (so. z.B. eine Umkehr des Sorgfaltsbeweises zulasten des Geschädigten; dazu sogleich) gemeint sind. Die Ausführungen haben gezeigt, dass die Haftungsnorm in ihrer Konzeption (enger Kontrollbegriff, Festhalten am Sorgfaltsbeweis) bereits jetzt

Medienmitteilung der RK-S vom 17. Oktober 2018: Kommission tritt auf den indirekten Gegenentwurf zur Konzernverantwortungsinitiative ein.

49 Stellvertretend für die Redundanz z.B. die zweimalige Erwähnung des Passus «Bestimmungen zum Schutz der Menschenrechte und der Umwelt». Eine Norm mit komplexen Inhalten wie den vorliegenden über zwei Sätze und zehn Zeilen zu erstrecken, sprengt zweifellos den Rahmen einer übersichtlichen und verständlichen Bestimmung. bescheiden ausfällt und im Vergleich zur Initiative deutliche Abstriche aufweist. Eine weitere Ausdünnung könnte somit einen Rückschritt hinter das geltende Recht bedeuten. Diese These beleuchte ich beispielhaft anhand des im Zuge dieser Abhandlung dargelegten Sorgfaltsbeweises:

3o Der Sorgfaltsbeweis ist wie erwähnt ein im Prinzip überkommenes Tatbestandselement (vgl. Kap. II.2.). Ich plädiere deshalb an sich dafür, darauf zu verzichten. Hält der Ständerat dennoch am Sorgfaltsbeweis fest, muss dieser zumindest vorbehaltlos beim Beklagten bleiben. Jenen Stimmen, welche die Beweislast im Rahmen von Art. 55 Abs. $1^{\text {bis }}$ E-OR statt dem Unternehmen nun der geschädigten Person auferlegen wollen,5o bleibt bei dieser Ausgangslage umso entschiedener zu entgegnen: Die Geschäftsherrenhaftung basiert auf dem Verständnis, dass sich der Geschäftsherr von einem schädigenden Fehlverhalten seiner Hilfsperson durch einen Sorgfaltsbeweis haftpflichtrechtlich distanzieren kann.51 Diesen Sorgfaltsbeweis - oder eben Entlastungsbeweis - hat systembedingt das Unternehmen $\mathrm{zu}$ erbringen. Vorliegend ist diese Beweislastverteilung umso vordringlicher, als der Möglichkeit eines unternehmerischen Sorgfaltsbeweises im Gesamtkonzept betont präventive Funktion zukommt (vgl. Kap. II.4. vorstehend).

31 Wer nun behauptet, der Entlastungsbeweis sei eine systemfremde unternehmerische Last, die der Unschuldsvermutung

50 Vgl. Hansueli Schöchli, Die Beweislast soll beim Kläger sein, NZZ online vom 28. Juni 2018.

${ }^{51}$ In diesem Sinne treffend auch SKMR/SIR (Fn. 43), Executive Summary, Rz. 21. 
widerspreche,52 irrt nicht nur zwischen Straf- und Haftpflichtrecht und verkennt das erwähnte Konzept der Geschäftsherrenhaftung. Er verschliesst die Augen vor massgeblichen Rechtstatsachen. So dürfte es dem Geschädigten kaum je möglich sein zu beweisen, dass das Unternehmen die gebotene Sorgfalt hat walten lassen, liegen doch die Beweise beim Unternehmen und sind diese wegen der fehlenden prozessualen Herausgabepflicht gegenüber dem Beklagten für Aussenstehende kaum je zugänglich.53 Der Geschädigte hätte gegebenenfalls nicht nur den Beweis des Schadens, des widerrechtlichen Fehlverhaltens der Tochtergesellschaft, den mitunter heiklen Beweis der «tatsächlich ausgeübten Kontrolle» sowie den notorisch schwierigen Beweis des Kausalzusammenhangs zu erbringen. 54 Er hätte darüber hinaus auch die kaum zu erbringende Verletzung der gebotenen Sorgfalt der Muttergesellschaft zu beweisen. Eine erfolgreiche Klage bliebe so faktisch unmöglich.

Die in dieser Deutlichkeit erkannte Notwendigkeit der konzeptuell vorgesehenen Beweislast muss, so meine ich, ihre Kraft auch gegenüber den im Kommissionsbericht zum Gegenentwurf erwähnten Zertifizierungen behalten.55 Soweit Unternehmen dereinst etwa Teile einer men-

52 So etwa Ehrat (Fn. 44).

$53 \mathrm{Zu}$ den konkreten Beweisschwierigkeiten wegen fehlender Herausgabepflichten der Gegenpartei eingehend Geisser (Fn. 23), Rz. 423 ff. In anderen Staaten wie Frankreich, England oder den Niederlanden bestehen hingegen grundsätzlich prozessuale Editionspflichten.

$54 \mathrm{Zu}$ den möglichen Beweisherausforderungen im vorliegenden Zusammenhang vgl. u.a. Hofstetter (Fn. 22), S. $240 \mathrm{ff}$.

55 Kommissionsbericht (Fn. 5), S. 6 (dort auch mit dem Hinweis auf eine entsprechende «Vermutung» der Anwendung der angewendeten Sorgfalt gemäss Art. 55 Abs. $1^{\text {bis }}$ E-OR). schenrechtlichen Sorgfaltsprüfung Revisionsstellen zur Prüfung vorlegen, darf nach meiner Auffassung ein Prüfbericht allenfalls die Basis für eine tatsächliche Vermutung der haftpflichtrechtlich gebotenen Sorgfalt bilden, nicht aber jene für eine gesetzliche Vermutung. ${ }^{6}$ Eine erfolgreiche Zertifizierung sollte also nicht zu einer Umkehr des Sorgfaltsbeweises zulasten des Geschädigten führen und ihm den Hauptbeweis auferlegen. Entschiede der Gesetzgeber anders, wäre das erwähnte Konzept der Geschäftsherrenhaftung auf den Kopf gestellt. Denn einer derart pauschalen, externalisierten «Checklisten-Prüfung» kann schlechterdings nicht die Fähigkeit zukommen, die haftpflichtrechtlich nach den «konkreten Umständen» 57 gebotene Sorgfalt eines Unternehmens zu bestimmen. Das UNO-Hochkommissariat für Menschenrechte spricht im vorliegenden Zusammenhang denn auch treffend von einer systemimmanenten «inappropriateness and unfairness of business enterprises seeking to raise due diligence defenses in cases where superficial scheck box> approaches to human rights due diligence might be used as a reference point.» 58

\section{Zusammenfassung}

33 Die vorstehenden Thesen lassen sich demnach wie folgt zusammenfassen:

(1) Die Geschäftsherrenhaftung ist wie andere funktional vergleichbare In-

${ }_{56}$ Zur Abgrenzung von tatsächlichen und gesetzlichen Vermutungen vgl. Peter Guyan, in: Basler Kommentar, Schweizerische Zivilprozessordnung, 3. Aufl., Basel 2017, N. 13 zu Art. 157 ZPO.

57 Vgl. statt vieler Rey/Wildhaber (Fn. 13), Rz. 1087; Fellmann/Kottmann (Fn. 11), Rz. 753 zum dahin gehenden Wortlaut von Art. 51 Abs. 1 OR (Fn. 6).

58 OHCHR (Fn. 37), Ziff. 29. 
stitute ein international allgegenwärtiges Haftungskonzept. Sie bildet die Grundnorm für typische Unternehmensrisiken. Ihr liegt die bestechende Rechtfertigung zugrunde, dass wer aus der Tätigkeit eines Anderen wirtschaftlichen Nutzen zieht, auch die damit verbundenen Risiken von Schädigungen Dritter tragen soll. Als solche ist diese Haftung in der Vergangenheit immer wieder Modell gestanden für richterliche Fortentwicklungen bis hin zu neuen Gesetzen.

(2) Indem sowohl die Konzernverantwortungsinitiative als auch der Gegenentwurf dem Unternehmen im Klagefall einen Entlastungsbeweis zur Verfügung stellen, bauen beide Vorlagen mit Art. 55 OR auf eine im internationalen Vergleich äusserst milde, unternehmensfreundliche Variante der Geschäftsherrenhaftung auf.

(3) Die Übertragung dieser Haftung oder vergleichbarer Institute auf Konzernverhältnisse folgt wiederum einem klaren in- und ausländischen Trend, der bereits im geltenden Recht angelegt ist. Eine Übertragung rechtfertigt sich durch den schlagenden Grundsatz, dass soweit herrschende Gesellschaften ihren Handlungsspielraum durch den Einsatz von abhängigen Gesellschaften ausweiten, sie auch für die Handlungen in diesem erweiterten Handlungsbereich haftpflichtrechtlich mitverantwortlich sind.

(4) Initiative wie Gegenentwurf bauen demnach auf einer sachgerechten und in ihrer schweizerischen Ausgestaltung zurückhaltenden Haftungs- norm auf. Beide Vorlagen erweitern den geltenden Haftungsrahmen nicht, sondern präzisieren ihn. Das gilt auch im vorliegend menschenrechtlichen Zusammenhang. Indem sich die in dieser Regelung geschützten Rechtsgüter auf «Leib und Leben oder Eigentum» beschränken, bleiben sie haftpflichtrechtlich im traditionellen Sinne eingegrenzt und im Privatrecht eingebettet.

(5) Die im Gegenentwurf verankerte Haftungsregelung fällt dabei auffallend einschränkend aus. Sowohl der Entscheid für den Entlastungsbeweis als auch jener für einen mehrfach eingeschränkten Kontrollbegriff führen zu einer sehr zurückhaltenden Haftungsnorm. Eine weitere Beschneidung dieser dünnen Vorlage so etwa mit einer systemfremden Umkehr des Sorgfaltsbeweises zulasten des Geschädigten - fiele hinter geltendes Recht zurück.

34 Ein Zusammenzug dieser Grundthesen führt zum Schluss, dass der indirekte Gegenentwurf den bestehenden Haftungsrahmen nicht erweitert. Die Schweiz wählt mithin eine Lösung, die im Einklang steht mit ihrer Rechtstradition. Dabei wählt die Schweiz eine im internationalen Vergleich zurückhaltende Lösung. Wenn unser Land in Nachachtung der UNO-Leitprinzipien für Wirtschaft und Menschenrechte mit dem Gesetzesentwurf allenfalls eine international aktive Rolle übernimmt, dann besteht diese lediglich in der Konkretisierung der bestehenden Haftungsgrundlagen und nicht in der Erweiterung dieser. 
35 In diesem Sinne sollte es der Schweiz als Rechtsstaat kontinentaleuropäischer Prägung zumindest ein Anliegen sein, der bereits im geltenden Recht angelegten Konzernorganisationhaftung im beschränkt menschenrechtlichen Zusammenhang einen gesetzlichen Grundrahmen zu geben. Damit blieben wichtige und de lege lata weitgehend ungeklärte Fragen nicht alleine dem weiten Ermessen richterlicher Rechtsfindung überlassen. Die entworfene Gesetzgebung kann in beschränktem Umfang nicht nur den Menschenrechtsschutz stärken, sondern dient vor allem der Rechtssicherheit. Dieser Befund schweizerischer Zurückhaltung bezieht sich auf materielles Haftpflichtrecht. Das Prozessrecht war nicht Gegenstand dieser Untersuchung. Unter Berücksichtigung des international notorisch beklagtenfreundlichen Zivilprozessrechts der Schweiz dürfte die Vorlage in einer Gesamtbilanz noch um Einiges bescheidener ausfallen. Von der mitunter befürchteten Klageflut ist bei nüchterner rechtlicher Betrachtung demnach nicht auszugehen. 